\title{
Aspects de la mise en œuvre d'un projet de langue mené par l'apprenant : la place de la sensibilisation
}

Joline Boulon

\section{(2) OpenEdition}

\section{Journals}

Édition électronique

URL : http://journals.openedition.org/asp/1894

DOI : 10.4000/asp.1894

ISBN : 978-2-8218-0384-8

ISSN : 2108-6354

\section{Éditeur}

Groupe d'étude et de recherche en anglais de spécialité

\section{Édition imprimée}

Date de publication : 1 octobre 2001

Pagination : 123-132

ISSN : 1246-8185

\section{Référence électronique}

Joline Boulon, «Aspects de la mise en œuvre d'un projet de langue mené par l'apprenant : la place de la sensibilisation », ASp [En ligne], 31-33 | 2001, mis en ligne le 12 novembre 2010, consulté le 20 avril 2019. URL : http://journals.openedition.org/asp/1894; DOI : 10.4000/asp.1894

Ce document a été généré automatiquement le 20 avril 2019.

Tous droits réservés 


\title{
Aspects de la mise en cuvre d'un projet de langue mené par l'apprenant : la place de la sensibilisation
}

\author{
Joline Boulon
}

\section{Introduction}

Dans l'une des composantes de Lyon 1 (l'IG2E - Institut de gestion de l'environnement et de l'écodéveloppement), nous avons offert un enseignement d'anglais présentant trois volets : un cours par semaine avec l'enseignant, un travail en autonomie au centre de ressources multimédia sur la précision (structures, formes, orthographe, etc.), et un projet personnel à réaliser en accord avec l'enseignant. C'est le dernier des trois volets, le travail en autonomie sur un projet personnel, qui est décrit dans cet article, sans pour autant faire abstraction des deux autres volets. Le projet personnel à réaliser est conçu à partir de plusieurs tâches ${ }^{1}$. Les étapes de chaque tâche à effectuer sont décrites en détail dans une collection de fiches que l'on appellera "fiches guides", accessibles aux apprenants tout au long du projet. De cette façon, les apprenants ont la possibilité de revenir autant qu'ils le souhaitent aux instructions. Ces fiches guides reflètent les souhaits de l'enseignant et sont en quelque sorte la médiation entre l'apprenant, l'enseignant et le projet personnel. Il y a déjà sur Internet de nombreux sites qui présentent ce type de fiches guides. Ils explicitent des activités à effectuer par l'apprenant d'une L22 2 . Néanmoins, ces sites ne semblent pas avoir les mêmes objectifs pédagogiques que ceux que pourraient avoir les enseignants de langue. Pour un enseignant, il est essentiel de réfléchir d'abord aux compétences à consolider pour ensuite concevoir des tâches qui les favoriseraient. En empruntant cette démarche, nous avons pu dégager, pour toutes les compétences, des critères de choix de tâches à créer. Ces critères de choix, qui nous ont permis de réaliser des fiches guides, proviennent notamment de nos recherches sur la sensibilisation à la L2 (Boulon 1999). 


\section{La sensibilisation}

Le rôle du processus psycholinguistique semble important à analyser pour mieux adapter des techniques pédagogiques aux techniques d'apprentissage. Skehan (1998) développe une description de ce processus à partir d'études variées. La figure 1 illustre bien le mécanisme, avec au centre le facteur dominant de l'apprentissage, ce qu'il appelle « noticing». En effet, pour mémoriser une information, il faut l'avoir remarquée auparavant.

Figure 1. Influences on noticing and components of working memory and long term memory (Skehan $1998: 57$ )

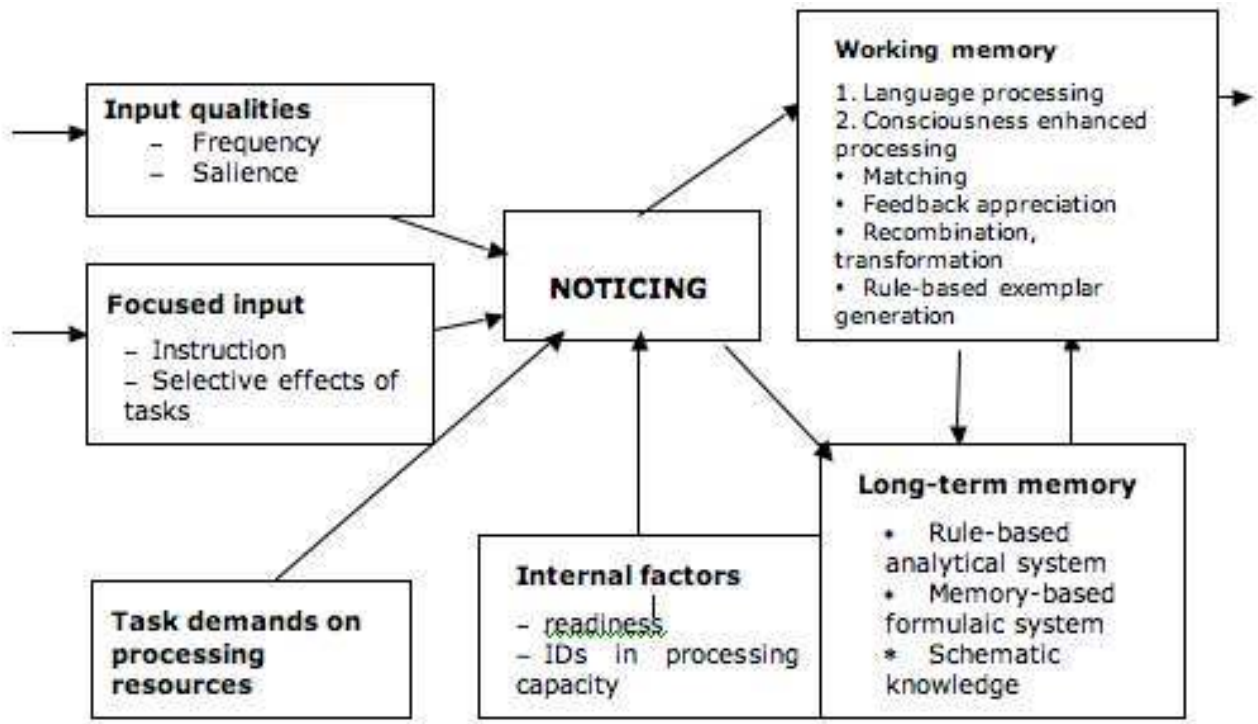

Les facteurs qui stimulent un apprenant sont variés :

- ${ }_{1}$ a fréquence d'une forme ou d'une lexie influence la capacité à les relever. Effectivement, plus une forme est répétée, plus elle aura de chances d'être observée par l'apprenant.

- L'enseignant peut focaliser l'attention des apprenants sur « l'input » (toutes les données que reçoit l'apprenant dans une situation de communication) pour faire constater les formes qui ne se répètent pas souvent.

- Certaines tâches peuvent faire ressortir différentes formes qui seront découvertes parce qu'elles sont inévitables.

- Les facteurs internes sont ceux qui sont régis uniquement par l'apprenant. Il doit être prêt à percevoir une forme, c'est-à-dire qu'il doit être à un niveau linguistique suffisant pour comprendre l'utilisation d'une structure non encore intériorisée.

- Les capacités à traiter les informations sont différentes pour chaque individu.

- Les rôles de la mémoire courte et de la mémoire longue sont aussi à prendre en compte. En effet, Skehan nous rappelle que l'acte de «percevoir » (noticing) s'effectue dans la mémoire courte (working memory) :

since the 'spotlight' consciousness which working memory provides is what is activated by the different influences upon noticing that we have covered. In this way, noticing becomes awareness and so the result of noticing becomes available for rehearsal, modification, and incorporation into long-term memory. (Skehan $1998: 51-52)$

C'est ce travail de sensibilisation à la L2 qui est prôné dans cet article. 


\section{Le cours à trois volets}

Ce cours a été conçu de façon à donner aux apprenants la possibilité d'adopter plusieurs stratégies d'apprentissage. Le cours avec l'enseignant est centré autour de l'expression orale et de la communication. En effet, c'est la forme d'enseignement la plus valorisante pour l'enseignant car aucune machine ne peut le remplacer dans ce rôle. Pendant ses cours, l'enseignant propose de nombreuses activités d'écoute, de prononciation et surtout de communication.

4 Le deuxième volet concerne l'étude des structures et des formes de la L2. Le travail s'effectue dans un centre de ressources en autoformation guidée. En effet, étant donné que chaque apprenant a ses propres lacunes, il n'a pas les mêmes besoins que les autres apprenants. Des tâches individualisées faites en autoformation guidée sont une bonne solution pour cette partie de l'apprentissage.

Enfin, le troisième volet, le projet personnel, est conçu comme moteur d'inspiration pour l'apprentissage de la langue. En effet, en choisissant son sujet, l'apprenant est plus motivé pour poursuivre les recherches inhérentes au projet. En même temps, il s'approprie les outils nécessaires pour améliorer ses compétences linguistiques. Plusieurs tâches sont demandées telles qu'un travail de bibliographie, une écoute de document oral et une transcription, des résumés de plusieurs documents écrits et une traduction, un travail de vocabulaire (mots clés et autres), un sommaire dans la L2, ainsi qu'un entretien avec des anglophones sur le sujet. Pour mener à bien ce projet, des fiches guides contenant la description détaillée de chaque tâche sont proposées à l'apprenant. Effectivement, en ayant de telles fiches, l'apprenant peut travailler à son rythme et n'a pas besoin de consulter l'enseignant à chaque étape du projet.

notre expérience montre que le projet personnel est difficile à gérer et pour l'enseignant et pour l'apprenant. L'enseignant veut un travail qui réponde à certains critères pédagogiques. Les apprenants, eux, ne tiennent pas toujours compte de ces critères. En proposant des fiches guides contenant plusieurs tâches avec des objectifs bien décrits, la compréhension de la part de l'apprenant est d'autant plus facilitée et les divergences arrivent à s'estomper quelque peu.

\section{Le projet personnel}

7 Le projet personnel a été conçu de façon à ce qu'il réponde à plusieurs questions didactiques. Il est important de fournir un travail sur plusieurs documents et types de documents dans la L2 pour éviter que l'apprenant ne voie que certains aspects, certaines structures, etc., et les fossilise. Mais il est aussi nécessaire de conduire l'apprenant à traiter un seul document à la fois, et à se concentrer sur une forme ou un aspect, car il n'est pas possible de se focaliser sur plusieurs aspects linguistiques simultanément (VanPatten cité dans Skehan 1998: 46-47). L'enseignant doit vérifier que toutes les compétences sont utilisées dans le projet car elles sont complémentaires. Enfin, le projet personnel doit permettre à l'apprenant de créer un fil conducteur dans son travail. Au lieu d'étudier plusieurs types de documents avec le seul but linguistique d'analyser plusieurs aspects de la langue, l'apprenant effectue un travail qui a un sens : il explore un sujet qui l'intéresse tout en se penchant sur l'aspect linguistique. 
8 La première année de la mise en place d'un enseignement à trois volets, les apprenants ont abordé le projet personnel de façon très individuelle avec très peu de contraintes. Notre souci était celui de motiver l'apprenant en lui proposant de choisir lui-même le sujet et la façon de traiter les données. Les études montrent que l'apprentissage est facilité quand l'apprenant est face à un besoin (Narcy 1997 : 60-65). Le projet personnel était conçu pour créer des besoins et donc améliorer l'apprentissage, il ne présentait que peu de contraintes : résumé des documents écrits et oraux, production d'une liste de vocabulaire, analyse des structures et des formes au choix. Les apprenants étaient très motivés par ce type de projet. En revanche, le résultat était mitigé : présentation des projets inacceptable pour un IUP (rapports rendus parfois sur feuilles de brouillon, parfois écrits à la main, parfois tout en français, etc.) ; sujets souvent traités de façon peu sérieuse (une étude sur la musique des Beatles était devenue uniquement une transcription de paroles, etc.) ; non respect des consignes (certains apprenants n'avaient pas «trouvé » de document oral, etc.). Les besoins que ce type de projet devait engendrer pour améliorer l'apprentissage ne se sont pas manifestés car les apprenants avaient préféré les éviter en créant des déviations (par exemple, certains résumés étaient rédigés entièrement au prétérit, ou avec des phrases très simples: sujet, verbe, objet). Donc, malgré leur enthousiasme pour ce type de travail, et malgré certains projets qui étaient, eux, excellents, il était indispensable de modifier les consignes.

a deuxième année, des consignes plus précises ont été conçues. Elles précisaient, pour chaque compétence, de nombreux types de documents à étudier. L'apprenant était obligé d'en choisir un certain nombre par compétence tout en se tenant à l'intérieur du sujet qu'il avait choisi. Des consignes claires pour la présentation finale étaient également fournies. Les résultats furent encore mitigés, mais meilleurs que l'année précédente. En effet, les présentations étaient devenues plus « universitaires ». Tout était dactylographié et présentable, en anglais et sur du papier propre. Certaines étaient même de qualité supérieure. Néanmoins, les apprenants se plaignaient, et parfois avec raison, que les documents oraux sur leur sujet d'étude n'existaient pas dans les différents centres de ressources. Ils étaient obligés de travailler sur des documents hors sujet, ce qui allait à l'encontre de la didactique mise en œuvre dans ce projet.

10 Pour tenter d'améliorer les performances l'année suivante, des fiches guides, devenues très explicites, ont été mises à la disposition des apprenants. La première tâche était de découvrir les différents documents qui existaient dans les diverses salles de ressources ainsi qu'à la bibliothèque universitaire pour pouvoir choisir un sujet en conséquence. Le choix du sujet était l'étape suivante, avec l'obligation de fournir à l'enseignant une bibliographie des sources avec une brève description de chaque document. Ces deux tâches ont permis d'éviter les problèmes survenus les années précédentes concernant les documents qui ne correspondaient pas au sujet.

11 Les tâches suivantes visaient à faire réfléchir les apprenants soit au sens des différents documents (travail de fluency dans la compréhension orale ou écrite, de résumé détaillé en L1 ou L2), soit aux différentes formes et structures contenues dans les documents (travail de précision, étude des temps utilisés, transcription des parties clefs des documents oraux, création de mots croisés pour les mots clefs du sujet, etc.)

C'est ce dernier canevas qui a apporté le meilleur résultat. En effet, les présentations orales des projets personnels étaient comparables à des communications faites par des professionnels. Les apprenants étaient à l'aise en anglais, leur souci étant de communiquer des données et des connaissances sur leur sujet. Dans les dossiers écrits, 
l'anglais était souvent assez soigné et, dans tous les cas, d'un niveau plus haut que les années précédentes. Les dossiers portaient des traces de véritable professionnalisme: souci de présentation, bibliographies, notes de bas de pages, annexes, recherches approfondies, etc. Car il ne faut pas oublier que la communication ne passe pas uniquement par la langue. Pour retrouver quels étaient les critères de choix à prendre en compte dans le développement de ces différentes tâches, nous avons analysé des résultats de certaines recherches (Ellis, Narcy, Skehan, etc.).

\section{Les critères de choix}

Face à un document oral ou écrit à analyser, l'apprenant explore tout d'abord le contenu. Skehan nous rappelle qu'un des principes soutenus par VanPatten dans le traitement de l'input est que l'apprenant traite d'abord le contenu : «Learners process input for meaning before they process it for form » (Skehan 1998: 46). Le travail sur la forme n'est pas possible sans avoir préalablement travaillé le sens. Il semble difficile de réaliser un travail de sensibilisation sur la forme dans un contexte de discussion, par exemple. En revanche, sur des documents de compréhension écrite ou orale, avec des activités de sensibilisation, il est possible, au stade de l'input, de travailler implicitement certaines formes pour améliorer la compréhension du document. «Implicit learning can be comprehension driven, provided that there is adequate focus on form in the input materials " (Doughty 1991 cité dans Skehan 1998: 64). Les premières tâches du projet, après celle de la bibliographie, conduisent donc l'apprenant à comprendre le sens d'un document et à lui faire percevoir certaines structures porteuses de sens.

Aussi, la sensibilisation à la forme est essentielle dans un deuxième temps car structures et formes ne s'apprennent pas uniquement en écoutant ou en lisant, ni en parlant ou en écrivant. Rod Ellis nous rappelle qu'une présentation explicite des formes donnée par l'enseignant n'est pas utile pour le discours (1997: 85). L'attention à la forme à travers une négociation du sens n'a pas porté ses fruits non plus (Ellis 1997: 50). Il faut que l'apprenant soit focalisé sur la forme de manière inductive, pour lui faire prendre conscience de la structure. Toutefois, cette focalisation doit être menée naturellement (Skehan 1998: 40). Nous proposons la sensibilisation à travers plusieurs tâches qui éveillent la curiosité à travers des activités de découverte.

Demander à l'apprenant de se focaliser sur certaines formes ou structures pour les placer au niveau conscient incite l'apprenant à fonctionner sur un système analytique, ce qui pourrait amener un changement à plus long terme. Plus l'apprenant se fonde sur ces processus, plus il s'appuiera sur sa mémoire à long terme pour la production, ce qui pourrait encourager l'apprenant à changer l'interlangue ${ }^{3}$ sous-jacente et à consolider ce changement (Skehan 1998: 57). Par conséquent, certaines tâches doivent amener les apprenants à analyser certaines structures ou formes. Toutefois, l'apprenant doit d'abord ressentir un besoin conscient de comprendre ces formes avant de remplir ces tâches. Des activités qui préconisent la sensibilisation aux structures de la L2 et qui amènent l'analyse de ces formes au niveau conscient de l'apprenant, sont sûrement plus bénéfiques à long terme que les activités qui entraînent l'apprenant à n'utiliser qu'une seule forme (Willis 1996 : 16).

16 Pour chaque compétence, il y a des critères de choix à intégrer avant de se lancer dans la conception de tâches. Pour la compréhension orale, il y a plusieurs aspects à analyser : la prononciation, l'intonation, les connaissances du sujet, la syntaxe, les formes et 
structures. Pour la production orale, les mêmes aspects peuvent être abordés mais de façon différente. Pour la compréhension écrite, il y a le vocabulaire, l'orthographe, la syntaxe, les formes et structures, les connaissances du sujet. Pour la production écrite, les mêmes aspects peuvent être abordés, mais de façon différente. Ces listes ne sont pas exhaustives. Même la communication interactive entre un apprenant et un enseignant d'anglais peut être traitée par des tâches et nécessite des critères de choix.

\section{Les tâches}

\subsection{La compréhension orale} travail de précision ou de compréhension globale. Les activités ${ }^{4}$ à l'intérieur de chaque tâche doivent comporter un but très précis (la compréhension globale, la reconnaissance d'un seul son, l'accent tonique, l'intonation, etc.). La sensibilisation signifie que chaque activité doit amener l'apprenant vers la découverte de l'objet même de l'activité. Par exemple, au lieu de faire remarquer à l'apprenant que les noms commençant par une voyelle sont précédés de «an» plutôt que « $a$ » sauf devant certains mots - « union, universal, university, European »- il serait préférable que l'activité lui demande de noter tous les mots précédés de « $a$ » et « an », puis d'en analyser le pourquoi (remarquera-t-il la consonne [j] de « union, universal, university, European»?). Des questions pourraient être posées pour diriger l'apprenant vers la découverte.

la forme, etc.). En effet, l'auditeur a tendance à écouter le fond avant de pouvoir se concentrer sur la forme. Dans nos fiches guides, par exemple, nous avons demandé à l'apprenant un résumé de quelques lignes pour chaque document qu'il compte utiliser pour mener à bien son projet, et ce dès la deuxième tâche, celle de la bibliographie. En organisant les tâches de cette façon, on permet à l'apprenant de compléter son travail de façon naturelle et sans frustration.

Certaines activités à l'intérieur des tâches doivent impérativement sensibiliser à la prononciation, étape par étape. En effet, certains sons sont perçus comme étant un allomorphe d'un phonème de la L1 alors qu'ils sont des phonèmes différents dans la L2 (par exemple, le $[\mathrm{r}]$ normand sur le bout de la langue n'est qu'un allomorphe du phonème /r/ français ; par contre le /i:/ anglais est un phonème et le /I/ en est un autre, bien qu'ils soient entendus par des francophones comme étant des allomorphes du même phonème / i/ en français.) Ainsi, les activités doivent amener l'apprenant à distinguer ces deux phonèmes avant même de lui demander de les prononcer.

D'autres activités doivent sensibiliser l'apprenant au "manque». Un francophone n'entend pas toujours les « petits mots insignifiants » tels que « that », «can », «but », « to ", etc. Ceci provoque souvent une incompréhension de la part de l'apprenant car ces mots ont une signification parfois indispensable dans la compréhension de la phrase.

\subsection{Production orale}

21 Les tâches de la production orale font suite à celles de la compréhension orale. Elles ont comme objectif pédagogique de sensibiliser l'apprenant à la production préparée, à la communication, à la prononciation, etc. Leurs activités doivent comporter un but très 
précis (fluency, production d'une structure, prononciation d'un son, accent tonique, intonation, etc.). Si l'apprenant dit: «is » pour " his » et « his » pour «is", les activités doivent être préparées de telle façon que l'apprenant soit amené lui-même à le remarquer. Dans ce cas, un travail en parallèle avec la compréhension orale serait bénéfique. L'apprenant doit découvrir ses propres erreurs avant d'amorcer le travail de correction.

Une tâche peut demander à l'apprenant de préparer un questionnaire sur le sujet de son projet personnel dans l'objectif d'interviewer un anglophone. Ensuite, l'apprenant est amené à rencontrer un anglophone et à enregistrer leur entretien. À partir de cet enregistrement, un grand nombre d'activités (de compréhension orale notamment) peuvent être envisagées.

\subsection{Compréhension écrite}

23 Les tâches ont comme objectif de sensibiliser l'apprenant à la lecture, que ce soit dans un travail de précision ou de compréhension globale. Les activités doivent comporter un but très précis (compréhension globale, orthographe d'un son, lexique, forme, structure, etc.). Mais au lieu de faire remarquer que le «have -en » dans tel document représente une conséquence des événements décrits, eux, au prétérit, il serait plus astucieux de demander à l'apprenant d'analyser la fonction des différents aspects et de lui demander d'en décrire (en L1) les utilisations.

Les tâches de compréhension doivent être premières car le lecteur a tendance à lire pour comprendre le fond avant de se concentrer sur la forme. L'apprenant peut rédiger un résumé de quelques lignes pour chaque document écrit qu'il choisit de lire pour mener à bien son projet avant de se pencher, par exemple, sur les structures. En organisant les activités de cette manière, l'enseignant permet à l'apprenant de compléter son travail sans frustration au niveau de la compréhension globale du document.

Certaines activités peuvent demander l'analyse d'une même structure dans des contextes différents. Un logiciel de concordance est utile dans ce cas. Dans le corpus de textes choisis par l'apprenant, il est possible de demander au logiciel de retrouver toutes les phrases contenant, par exemple, une préposition. De cette façon, l'apprenant peut effectuer une analyse des différentes utilisations de cette préposition en repérant les différentes notions qu'elle comporte.

\subsection{La production écrite}

Les tâches de la production écrite s'effectuent à la suite de celles de la compréhension écrite. Elles ont comme objectif de sensibiliser l'apprenant à la production de résumés, d'articles, d'instructions, etc. Les activités doivent comporter un but très précis (réalisation d'un résumé, apprentissage de l'orthographe de vocabulaire précis, étude du lexique, etc.). Cependant, au lieu de se voir expliquer la forme d'un abstract en anglais, l'apprenant en lit plusieurs dans le but de retrouver ce qu'ils ont en commun. Il doit ensuite décrire la structure de l'abstract avant de tenter la rédaction.

Il est important de souligner ici que chaque activité ne doit comporter qu'un seul objectif. Si l'objectif est de rédiger un abstract, l'enseignant doit, par exemple, exclure dans la notation, les erreurs d'orthographe, (ce qui ne veut pas dire qu'il ne faut pas les signaler). 
Dans une rédaction qui a pour but de sensibiliser l'apprenant à l'orthographe, c'est cet aspect qui sera noté, et une erreur de structure sera simplement signalée.

Bien entendu, ces exemples sont très loin d'être exhaustifs, le but étant uniquement d'illustrer nos différents propos. Il est à noter que ce type de travail en autoformation guidée prend énormément de temps à faire pour l'apprenant, à concevoir et à corriger pour l'enseignant. Un quart d'heure passé avec quelques apprenants lorsqu'ils préparent une activité en petits groupes peut être bénéfique. En effet, l'apprenant en profite pour présenter ses activités complétées au fur et à mesure qu'il les termine, ce qui permet à l'enseignant de vérifier tout au long de l'année que les tâches sont bien comprises. Ainsi, la correction des projets s'étale sur le semestre ou l'année.

\section{Fiches guides}

Le rôle des fiches guides est de présenter toutes les tâches et leurs activités -choisies et organisées par l'enseignant- pour permettre aux apprenants d'accomplir leur projet personnel avec la plus grande efficacité, de façon individualisée, et sans avoir recours à l'enseignant à la fin de chaque activité. En plus des tâches, les fiches doivent présenter la forme que prend le projet, autant pour une éventuelle présentation orale que pour un éventuel dossier écrit. Elles ne doivent pas faire abstraction des détails, tels que les dates des présentations, le nombre de pages exigé par l'enseignant pour les différentes parties écrites, les horaires d'ouverture des différents centres de ressources...

La forme des fiches guides peut varier. En dehors d'une forme classique papier, elles peuvent être présentées sur Internet avec plusieurs liens, ou sur le serveur du centre de ressources. Un logiciel tel que «PowerPoint " (logiciel de Microsoft) s'avère intéressant pour ce type de présentation. Selon le nombre de tâches que l'enseignant estime nécessaires, les fiches guides peuvent être brèves ou assez étoffées.

\section{Conclusion}

Dans l'enseignement à trois volets que nous avons mis en place, le projet personnel sert à motiver l'apprenant car c'est lui qui choisit le sujet d'étude. Le projet demande à l'apprenant de consolider toutes les compétences linguistiques. Nous avons donc conçu des tâches à accomplir. Les activités à l'intérieur de chaque tâche ont un objectif précis et doivent sensibiliser l'apprenant plutôt que lui présenter des données. La médiation entre les souhaits de l'enseignant et ce projet personnel se trouve dans les fiches guides qui développent clairement et explicitement chaque tâche à réaliser. Ces fiches permettent à l'apprenant de mener à bien son projet personnel. En effet, l'apprenant doit savoir ce que l'enseignant attend de son projet, connaître les différents éléments du dossier, comprendre comment présenter les différents éléments du dossier ainsi que le projet final. Dans un tel contexte, grâce aux fiches guides, il est possible d'inclure des exigences à propos du contenu, comme des exigences beaucoup plus pointues sur l'analyse de différents aspects de la langue et du vocabulaire se trouvant dans les documents que l'apprenant avait sélectionnés auparavant. Pour mettre en place ce cours, nous nous sommes fondée sur les recherches actuelles dans les domaines de la cognition, notamment vue par Skehan. Il faut que les tâches créent un besoin, ce qui amène l'apprenant à remarquer un point, une structure. Cette sensibilisation lui permet 
d'analyser le point perçu, et évite à l'apprenant de se retrouver face à des explications qui l'ennuient, ne le concernent pas, qu'il ne comprend pas, etc. Créées en fonction de ces critères, les tâches doivent amener l'apprenant non seulement à terminer son projet personnel, mais à renforcer ses compétences linguistiques en L2.

\section{BIBLIOGRAPHIE}

Boulon, Joline. 1999. «Rôle et statut de la sensibilisation linguistique sur multimédia dans l'enseignement de l'anglais dispensé à des étudiants spécialistes d'autres disciplines ». Thèse de doctorat, Université Lyon 3.

Doughty, C. 1991. «Second language instruction does make a difference: evidence from an empirical study on SL relativization ». Studies in Second Language Acquisition 13, 431-69.

Ellis, Rod. 1997. SLA Research and Language Teaching. Oxford : Oxford University Press.

Narcy, Jean-Paul. 1997. « Vers une pratique théorisée et humaniste ». In Ginet A. et al. (dir.), Du Laboratoire de langues à la salle de cours multi-média. Paris : Nathan, 45-90.

Selinker, L. 1972. «Interlanguage ». IRAL 10, 209-31.

Skehan, Peter. 1998. A Cognitive Approach to Language Learning. Oxford : Oxford University Press.

VanPatten, B. 1996. Input Processing and Grammar Instruction. New York : Ablex.

Willis, Jane. 1996. A Framework for Task-Based Learning. Harlow : Longman.

\section{NOTES}

1. Dans cet article, une tâche correspond à la signification donnée par Willis : "tasks are always activities where the target language is used by the learner for a communicative purpose (goal) in order to achieve an outcome » $(1996: 23)$.

2. Par exemple: International Tandem Network http://www.slf.ruhr-uni-bochum.de/email/ idxeng00.html, WebQuest Assignment <http://www.d.umn.edu/ hrallis/courses/5413su05/ assignments/webquest.html>.

3. Le nom donné par Selinker aux connaissances langagières en voie de développement propre à chaque apprenant de L2 (1972).

4. Une tâche est composée de plusieurs activités que l'apprenant doit effectuer pour accomplir la tâche. 


\section{RÉSUMÉS}

Nous décrivons dans cette étude divers aspects de la mise en œuvre d'un projet de langue mené par l'apprenant, dans le cadre d'un enseignement intégré d'anglais pour spécialistes d'autres disciplines à l'université Lyon 1 . Pour mener à bien son projet, l'apprenant doit être conduit à découvrir et analyser par lui-même les différentes formes et structures. Ceci est rendu possible par la sensibilisation construite par un dispositif de fiches guides décrivant des tâches et activités précisément adaptées à l'objectif.

At the University of Lyon 1, an English course for specialists in disciplines other than English incorporated a personal project conceived by the learner, yet in keeping with the teacher's objectives. For the learner to work effectively, he had to be helped to discover and analyse for himself the various forms and structures involved. The teacher therefore had to create clear and explicit guidelines describing the different tasks and activities to be tackled and inviting him to improve his language and communication skills.

\section{INDEX}

Keywords : language awareness, meaning, noticing, task

Mots-clés : fond, perception, sensibilisation, tâche

\section{AUTEUR}

\section{JOLINE BOULON}

Joline Boulon est PAST à l'université Claude Bernard Lyon 1. Elle a soutenu une thèse sur la sensibilisation à la L2 grâce au multimédia dans l'apprentissage de l'anglais, sous la direction de M. Clay, à l'Université Jean Moulin Lyon 3. Elle poursuit ses recherches sur les outils qui mettent en œuvre la sensibilisation à la L2 dans l'apprentissage des langues. boulon@univ-lyon1.fr 